\title{
Emergence of Population Bursts from Simultaneous Activation of Small Subsets of preBötzinger Complex Inspiratory Neurons
}

\author{
Kaiwen Kam, ${ }^{1 \star}$ Jason W. Worrell, ${ }^{1 \star}$ Cathie Ventalon, ${ }^{2}$ Valentina Emiliani, ${ }^{2}$ and Jack L. Feldman ${ }^{1}$ \\ ${ }^{1}$ Systems Neurobiology Laboratory, Department of Neurobiology, David Geffen School of Medicine, University of California Los Angeles, Los Angeles, \\ California 90095-1763, and 'Wavefront-Engineering Microscopy Group, Neurophysiology and New Microscopies Laboratory, CNRS UMR 8154, INSERM \\ U603, University Paris Descartes, 75006 Paris, France
}

During rhythmic movements, central pattern generators (CPGs) trigger bursts of motor activity with precise timing. However, the number of neurons that must be activated within CPGs to generate motor output is unknown. In the mammalian breathing rhythm, a fundamentally important motor behavior, the preBötzinger Complex (preBötC) produces synchronous population-wide bursts of activity to control inspiratory movements. We probed mechanisms underlying inspiratory burst generation in the preBötC using holographic photolysis of caged glutamate in medullary slices from neonatal mice. With stimulation parameters determined to confine photoactivation to targeted neurons, simultaneous excitation of 4-9 targeted neurons could initiate ectopic, endogenous-like bursts with delays averaging $255 \mathrm{~ms}$, placing a critical and novel boundary condition on the microcircuit underlying respiratory rhythmogenesis.

\section{Introduction}

Breathing is a vital behavior regulated by hindbrain neural circuits that generate near synchronous bursts of action potentials in populations of neurons that ultimately drive inspiratory and expiratory movements. To adjust respiratory muscle activity when metabolic demand changes or during production of critical reflexes such as coughing or gagging, these hindbrain neural circuits must rapidly change timing through temporal control of burst initiation. The preBötzinger Complex (preBötC), a bilateral medullary population of $\sim 1000$ neurons/side, is essential for generating inspiratory bursts in vitro and in vivo (Feldman and Del Negro, 2006; Tan et al., 2008; Feldman et al., 2013). Inspiratory bursts can be initiated by injection of glutamate analogs into the ventral respiratory group in vivo (McCrimmon et al., 1986) or the preBötC in vitro (Del Negro et al., 2009), which asynchronously excite an unknown number of neurons. We hypothesized that rapid changes in burst timing necessary for lability in breathing rhythm can result from excitation and propagation of activity from a few preBötC neurons. Thus, we tested whether simultaneous suprathreshold excitation of a small number of preBötC neurons during the quiescent interburst interval could trigger a premature, i.e., ectopic, inspiratory burst.

\footnotetext{
Received Sept. 25, 2012; revised Dec. 17, 2012; accepted Dec. 20, 2012.

Author contributions: K.K., J.W.W., C.V., V.E., and J.L.F. designed research; K.K., J.W.W., and C.V. performed research; K.K. and J.W.W. analyzed data; K.K., J.W.W., C.V., V.E., and J.L.F. wrote the paper.

This work was supported by grants from the U.S. National Heart, Lung, and Blood Institute and National Institute of Neurological Disorders and Stroke. We thank David Sherman for assistance with immunohistochemistry and all members of the Feldman and Emiliani laboratories for helpful discussion.

*K.K. and J.W.W. contributed equally.

The authors declare no competing financial interests.

Correspondence should be addressed to Jack Feldman at the above address. E-mail: feldman@ucla.edu.

DOI:10.1523/JNEUROSCI.4574-12.2013

Copyright $\odot 2013$ the authors $\quad 0270-6474 / 13 / 333332-07 \$ 15.00 / 0$
}

Simultaneous excitation of multiple neurons cannot be accomplished with sequential techniques, e.g., acousto-optical or galvanometer deflectors, for photostimulation with a single laser beam (Zahid et al., 2010). To identify the inspiratory burst initiation threshold, we used holographic photolysis of MNIglutamate (a caged glutamate compound; Lutz et al., 2008; Zahid et al., 2010) to simultaneously induce action potential firing in several inspiratory-modulated preBötC neurons in medullary slices generating a respiratory-related rhythm.

\section{Materials and Methods}

Slice preparation and electrophysiology. Experiments followed European Union and Institutional Guidelines of the Care and Use of Laboratory Animals (Council Directive 86/609 EEC) and protocols approved by the Office for the Protection of Research Subjects (University of California Animal Research Committee). We cut 550- $\mu$ m-thick transverse medullary slices with preBötC at the rostral surface from neonatal C57BL/6 mice (postnatal days 0-5) of either sex (Smith et al., 1991; Ruangkittisakul et al., 2011). To identify inspiratorymodulated neurons, slices were loaded with $60 \mu \mathrm{m}$ Oregon Green BAPTA-1 AM (Invitrogen) for 30-60 min (Mukamel et al., 2009).

Slices were perfused with $27^{\circ} \mathrm{C}$ ACSF at $2.5 \mathrm{ml} / \mathrm{min}$ in a $0.5 \mathrm{ml}$ chamber and mounted rostral side up in a fixed-stage microscope (Olympus BX50WI). ACSF contained (in mM): $124 \mathrm{NaCl}, 3 \mathrm{KCl}, 1.5 \mathrm{CaCl}_{2}, 1$ $\mathrm{MgSO}_{4}, 25 \mathrm{NaHCO}_{3}, 0.5 \mathrm{NaH}_{2} \mathrm{PO}_{4}$, and 30 D-glucose, equilibrated with $95 \% \mathrm{O}_{2}$ and $5 \% \mathrm{CO}_{2}\left(27^{\circ} \mathrm{C}, \mathrm{pH}=7.4\right)$. A recycling perfusion system $(5-10 \mathrm{ml})$ allowed continuous circulation of MNI-glutamate $(500 \mu \mathrm{M}$, Tocris Bioscience). Extracellular potassium was raised to $9 \mathrm{~mm}$, and respiratory motor output was recorded from a XIIn or XII motor nucleus using suction electrodes and a MultiClamp 700B (Molecular Devices), filtered at $2-4 \mathrm{kHz}$, digitized at $10 \mathrm{kHz}$, full-wave rectified, and smoothed. Digitized data were analyzed using custom procedures written for IgorPro (Wavemetrics) and Matlab 2010a (The Mathworks). Before each experiment, we monitored the slice preparation 
for $30 \mathrm{~min}$ to ensure that XIIn frequency and magnitude reached steady state. In select experiments, tetrodotoxin ( $1 \mu \mathrm{M}$; Tocris Bioscience) was bath applied.

Whole-cell and loose-patch recordings were performed using a MultiClamp 700B. Intracellular solution contained (in mM): $135 \mathrm{KG}-$ luconate, 1.1 EGTA, $5 \mathrm{NaCl}, 0.1 \mathrm{CaCl}_{2}, 10$ HEPES, $2 \mathrm{MgATP}$, and 0.3 $\mathrm{Na}_{3}$ GTP. In whole-cell current-clamp recordings, membrane potentials were brought to $-70 \mathrm{mV}$ using a negative bias current. In voltage clamp, holding potential was $-65 \mathrm{mV}$. For loose-patch recordings, action potential (AP) shape was monitored to detect stability of the patch. For whole-cell recordings, experiments in which variations in series and input resistance exceeded the initial value by $25 \%$ were discarded.

Fluorescence imaging. For $\mathrm{Ca}^{2+}$ imaging, excitation light $(488 \mathrm{~nm} ; 10 \mathrm{~nm}$ bandwidth) was provided by a $75 \mathrm{~W}$ xenon lamp passing through a monochromator (Optoscan, Cairn Research), and fluorescence images were collected with a 12-bit cooled $\left(-30^{\circ} \mathrm{C}\right) \mathrm{CCD}$ camera (CoolSNAP HQ2, Roper Scientific). Emission wavelengths were obtained using a HQ535/50M filter (Chroma Technology). MetaMorph (Molecular Devices) was used to acquire (10 Hz, $50 \mathrm{~ms}$ exposure time) and store images for analysis. Background fluorescence was subtracted, and $\mathrm{Ca}^{2+}$ responses were expressed as relative changes in fluorescence $(\Delta F / F)$.

Either Cell Sort 1.0 or a program employing JBoost was used for automated detection of time-varying fluorescent signals and inspiratory preBötC neurons (Mukamel et al., 2009; Valmianski et al., 2010). ROIs with inspiratory-modulated $\mathrm{Ca}^{2+}$ signals were targeted for holographic photolysis. Neuronal signals were distinguished from presumptive astrocytic fluorescence rises, which were larger, slower, and not time locked to XIIn output (Ruangkittisakul et al., 2009).

Immunohistochemistry and histology. Procedures for immunohistochemistry were described previously (Tan et al., 2010). Fortymicrometer-thick transverse brainstem sections were cut using a freezing microtome and incubated in primary antibodies (rabbit anti-SST-14, Peninsula Laboratories, 1:500; and mouse anti-neuronal nuclei, NeuN; Millipore Bioscience Research Reagents ; 1:500) for $24 \mathrm{~h}$ at $25^{\circ} \mathrm{C}$. After washes, primary antibodies were detected using the following specific secondary antibodies (dilution 1:250): Alexa Fluor 568-conjugated donkey anti-rabbit and Alexa Fluor 488-conjugated donkey anti-mouse (Invitrogen). High resolution Z-stack images taken at $2 \mu \mathrm{m}$ intervals were acquired on a confocal microscope (LSM510 META, Carl Zeiss). Neuronal density was determined by dividing the number of NeuN-positive neurons within a $100 \mu \mathrm{m}$ radius circle ventral to nucleus ambiguus that contained SSTpositive neurons, which define the preBötC (Tan et al., 2010), by the cylindrical volume.

Holographic photolysis. The holographic microscope was mounted around an epifluorescence upright microscope (BX50WI; Olympus). We used a $405 \mathrm{~nm}$ diode laser (CUBE 405-100; Coherent) for uncaging combined into the microscope with a dichroic beamsplitter (425DCXR; Chroma Technology). The output beam was expanded $12.5 \times$ to match the input window of a liquid crystal spatial light modulator (LC-SLM, X10468; Hamamatsu) that operated in reflection mode. A telescope $\left(f_{1}=600 \mathrm{~mm}, f_{2}=200 \mathrm{~mm}\right)$ imaged the plane of the SLM to the rear aperture of a water immersion objective (Olympus) with infrared coating (UMPLFLN20XW; numerical aperture, 0.5). Some experiments were performed with the Phasor spatial light modulator (SLM) system (Intelligent Imaging Innovations). An iterative Fourier transform algorithm, implemented into customdesigned software (Lutz et al., 2008) or Slidebook 5 (Intelligent Imaging Innovations), computed the phase pattern on the SLM corresponding to the desired distribution of light intensity at the focal plane of the objective. A blocker was inserted in the intermediate Fourier plane to block the unmodulated light component (zero order spot) and replicate patterns (Golan et al., 2009).

Statistical analysis. Standard parametric tests were used to determine statistical significance. Student's $t$ test and a one-way ANOVA, followed by Tukey-Kramer analysis, were used when appropriate. Data are presented as mean \pm SEM. Statistical significance was set at $p<0.05$.
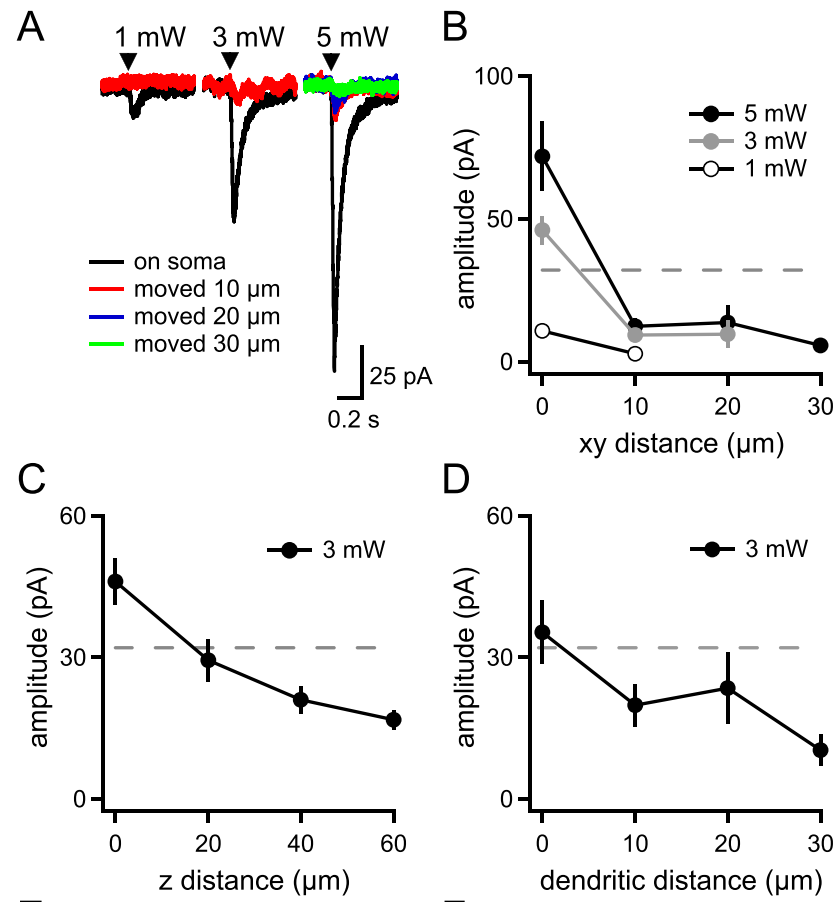

E

$\mathrm{D}$

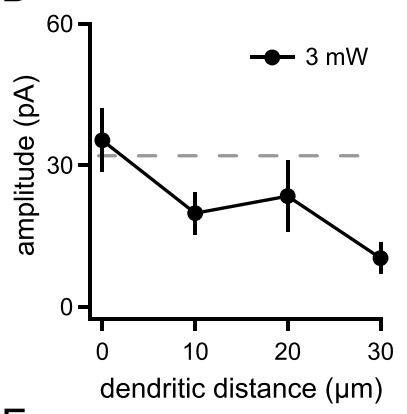

$\mathrm{F}$
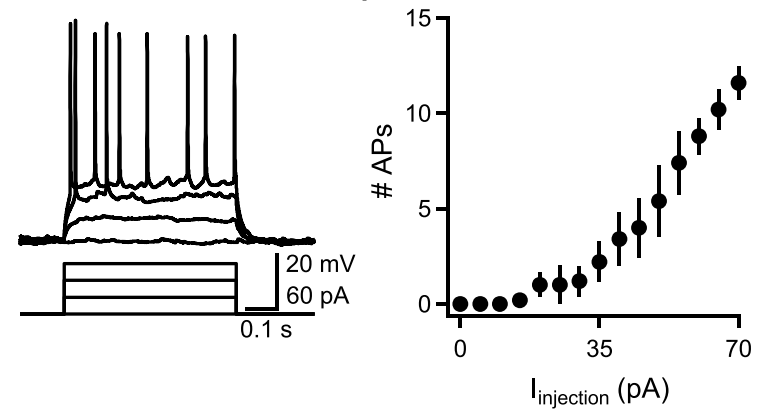

Figure 1. Responses of single preBötC neurons to holographic photostimulation. $\boldsymbol{A}$, Holographic photostimulation (black triangle) elicits glutamate-evoked currents in a single neuron recorded in tetrodotoxin $(1 \mu \mathrm{m})$. Representative inward currents increase in amplitude as a function of laser power, but decrease with xy distance from the soma. $\boldsymbol{B}-\boldsymbol{D}$, Glutamate-evoked current amplitude as a function of laser power and $x y$ distance of the spot from the soma ( $n=$ $4-19)(\boldsymbol{B}), z$ distance of the spot from the soma $(n=6)(\boldsymbol{C})$, and distance of the spot from the soma distally along a primary dendrite $(n=6)(\boldsymbol{D})$. Dotted lines in $\boldsymbol{B}-\boldsymbol{D}$ represent rheobase. $\boldsymbol{E}$, Representative preBötC inspiratory neuron responses to injected currents. $\boldsymbol{F}$, Number of APs elicited by current steps $(n=5)$.

\section{Results}

One to nine neurons were targeted using a spatial light modulator to shape a $405 \mathrm{~nm}$ laser beam into $10 \mu \mathrm{m}$ diameter somacentered spots. Because uncaged glutamate can diffuse from the site of photolysis to depolarize nontargeted neurons, we first characterized the lateral and axial resolution of photoactivation to determine stimulation parameters that limited excitation to the targeted neuron. Whole-cell patch-clamp recordings of preBötC neurons were performed in the presence of tetrodotoxin (TTX; $1 \mu \mathrm{M}$ ), while the distance between soma and laser spot was varied. Five $800 \mu$ s pulses $(200 \mathrm{~Hz})$ of a single $3 \mathrm{~mW}$ somacentered laser spot elicited an inward current in the targeted neuron of $46 \pm 5 \mathrm{pA}$ (Fig. $1 A, B ; n=19$ ). When the spot was displaced $10 \mu \mathrm{m}$ in the slice $(x y)$ plane, the current was reduced by $80 \%$ to $9.3 \pm 2.1 \mathrm{pA}(n=6$; Fig. $1 A, B)$. Only currents elicited by soma-centered spots would be sufficient to produce APs as rheobase, i.e., the threshold for AP generation, was $32 \pm 7 \mathrm{pA}$ $(n=5$; Fig. $1 E, F)$. 
A

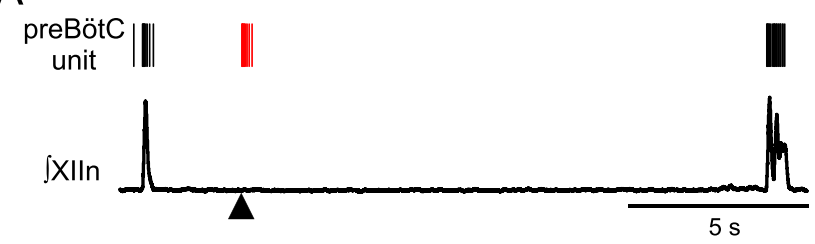

endogenous

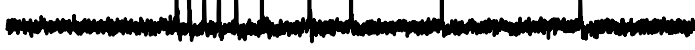

evoked

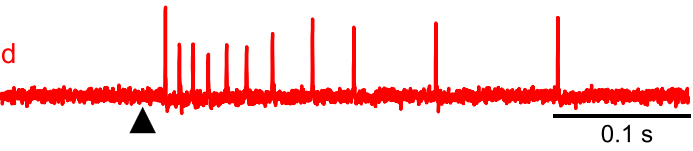

B

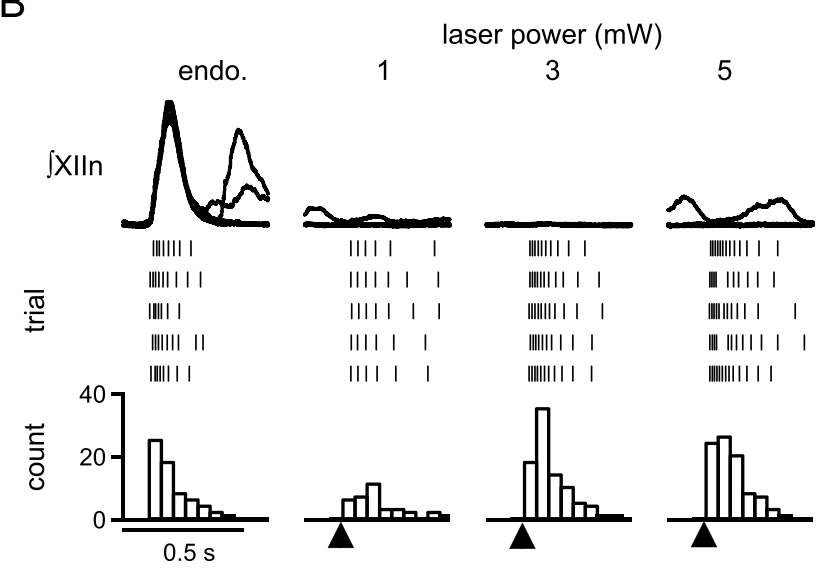

C
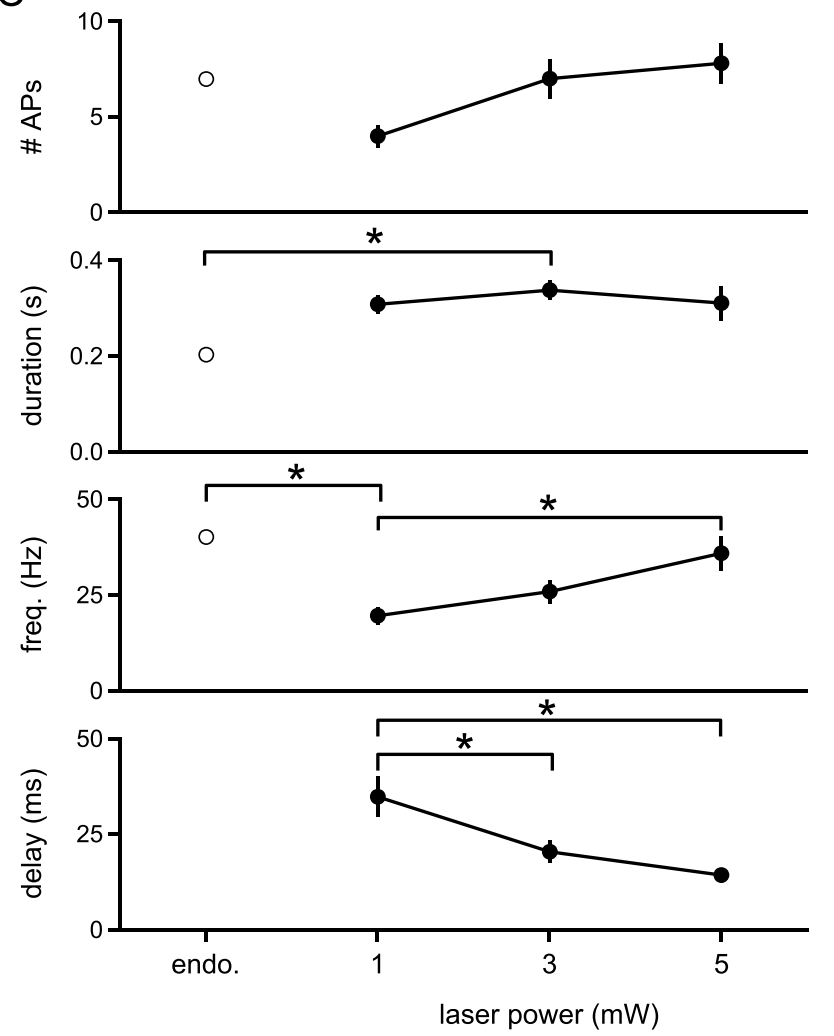

Figure 2. Cell-attached recordings of preBötC neuron firing patterns in response to photostimulation of the recorded neuron. $\boldsymbol{A}$, Top, XIIn recording and representative preBötC inspiratory neuron firing pattern at baseline (endogenous burst, black raster) and in response to a 3 $\mathrm{mW}$ laser spot targeted to soma of recorded neuron (black triangle; red raster; $5 \times 800 \mu \mathrm{s}$
The holographic pattern had an effective $z$-axial length \pm $18 \mu \mathrm{m}$, the theoretical full-width at half-maximum, relative to the focal plane. Given that targeted neurons were superficial, neurons within $\sim 18 \mu \mathrm{m}$ below the focal plane, comprising a cylindrical volume of $1400 \mu \mathrm{m}^{3}$, could also be directly illuminated. To determine the spatial density of preBötC neurons, NeuN-positive cell bodies were counted in a region marked by SST staining ventrolateral to nucleus ambiguus (Tan et al., 2008 ) and divided by the volume of the section yielding an average density of 0.0004 neurons $/ \mu \mathrm{m}^{3}$ (data not shown; $n=$ $6)$. Based on this spatial density, the illumination volume would only intersect $0.50 \pm 0.04$ neuronal cell bodies $(n=6)$.

To validate the illumination volume empirically, preBötC neuron responses to a $10 \mu \mathrm{m}, 3 \mathrm{~mW}$ photoactivation spot were recorded in $1 \mu \mathrm{M}$ TTX in whole-cell patch clamp as the focal plane was raised. Raising the focal plane $20 \mu \mathrm{m}$ reduced the current by $46 \%$ from $46 \pm 5 \mathrm{pA}(n=19)$ to $29.4 \pm 4.5 \mathrm{pA}(n=$ 6 ), below rheobase and consistent with the theoretical axial attenuation of the holographic beam (Fig. 1C). When the focal plane was raised $40 \mu \mathrm{m}$, current decreased further to $21 \pm 3$ pA $(n=6)$, likely representing a combination of glutamate diffusion and attenuated axial illumination below halfmaximum intensity (Fig. 1C). Thus, laser power up to $3 \mathrm{~mW}$ could trigger APs only in the one targeted neuron directly illuminated by a holographic beam, confirming the theoretical calculation. Neurons outside this beam should not reach rheobase, and any glutamate-evoked response would fail to propagate further.

Photolyzed glutamate could also depolarize dendrites passing through the illumination volume. To determine the effect of a holographic spot on dendrites, glutamate-evoked depolarization was measured in preBötC neurons with whole-cell patch clamp in $1 \mu \mathrm{M}$ TTX as a $10 \mu \mathrm{m}, 3 \mathrm{~mW}$ spot was moved in $10 \mu \mathrm{m}$ steps along a visible primary dendrite. The glutamate-evoked response to photostimulation of a spot displaced $10-30 \mu \mathrm{m}$ off the soma, but along a dendrite (Fig. $1 D$ ), was below rheobase, so stimulation of dendrites could not produce APs.

To determine the suprathreshold response of neurons to holographic photolysis of MNI-glutamate, firing patterns of inspiratory preBötC neurons were recorded in loose patch configuration. Respiratory rhythm in slices consisted of periodic inspiratory XIIn bursts (period: $4.5 \pm 0.6 \mathrm{~s} ; n=7$ ). MNIglutamate $(500 \mu \mathrm{M})$ added to the bath did not significantly change burst properties when compared to control (normalized to control: amplitude $1.05 \pm 0.09, t_{(12)}=-0.24, p=0.8$, halfwidth $=0.9 \pm 0.04 ; t_{(12)}=1.08, p=0.3$, area $=0.96 \pm 0.09$; $t_{(12)}=0.02, p=1$, period $\left.=4.2 \pm 0.4 \mathrm{~s} ; t_{(12)}=0.44, p=0.7 ; n=7\right)$. Endogenous inspiratory bursts in preBötC neurons consisted of

\footnotetext{
$\leftarrow$

pulses, $200 \mathrm{~Hz}$ ). Bottom, Firing patterns shown at top are expanded. Evoked firing pattern in response to laser does not exceed the frequency of firing during a normal endogenous burst. $\boldsymbol{B}$ Simultaneous recordings of XIIn (top) and suprathreshold responses to targeted photostimulation of the recorded neuron (black triangle) as a function of laser power $(5 \times 800 \mu$ s pulses, 200 $\mathrm{Hz}$; middle, bottom). Representative rasters of laser-evoked peristimulus spike times for 5 runs (middle) and peristimulus time histograms (bottom) are comparable to firing patterns elicited during an endogenous burst (endo.). C, Number of APs, duration of response, frequency, and time to first AP after stimulus $(5 \times 800 \mu$ s pulses, $200 \mathrm{~Hz})$ compared to firing patterns during endogenous bursts (endo.) and as a function of laser power (AP number: one-way ANOVA, $F_{(3,40)}=2.82, p=0.05$; duration of response: $F_{(3,39)}=3.13$, endo. vs $3 \mathrm{~mW}, p=0.03$; frequency: $F_{(3,39)}=6.68$, endo. vs $1 \mathrm{~mW}, p=0.002,1 \mathrm{mWvs} 5 \mathrm{~mW}, p=0.008$; time to first AP: $F_{(2,29)}=8.41,1 \mathrm{~mW}$ vs $3 \mathrm{~mW}, p=0.001,1 \mathrm{mWvs} 5 \mathrm{~mW}, p=0.02 ; n=11$ neurons in 8 slices $)$. ${ }^{*} p<0.05$.
} 
multiple APs, sometimes preceding but always coincident with XIIn bursts (AP number/burst $=7.0 \pm 0.1$, duration $=202 \pm 14 \mathrm{~ms}$, frequency $=40.1 \pm 1.0 \mathrm{~Hz} ; n=11$ neurons in 8 slices; Fig. 2). In some cases, sporadic firing during the interburst interval was observed (frequency $=3.7 \pm 0.1 \mathrm{~Hz}$ ). Stimulating single neurons during the interburst interval using the laser parameters characterized above (1-3 mW/spot, $5 \times 800 \mu \mathrm{s}, 200 \mathrm{~Hz}$; Fig. 1), produced bursts of APs that did not exceed the frequency of endogenous inspiratory bursts ( $3 \mathrm{~mW}$ stimulated activity: AP number/stimulus $=7.0 \pm 1.0$, duration $=337 \pm 21 \mathrm{~ms}$, frequency $=25.9 \pm 3.1 \mathrm{~Hz}$; delay to first $\mathrm{AP}=20 \pm 3 \mathrm{~ms} ; n=11$ neurons in 8 slices; Fig. $2 \mathrm{~B}, C)$. Stimulation of a single, arbitrarily chosen, inspiratory-modulated preBötC neuron with these parameters did not produce an ectopic XIIn burst ( $n=11$ neurons in 8 slices; Fig. $2 A, B$; Fig. $3 A$ ).

Next, we photostimulated one to nine inspiratorymodulated preBötC neurons simultaneously during the interburst interval to determine the threshold to initiate an ectopic XIIn burst. In slices loaded with the $\mathrm{Ca}^{2+}$ indicator dye Oregon Green BAPTA 1-AM, inspiratory preBötC neurons were identified by rhythmic fluorescence signals time-locked with XIIn bursts (Fig. 3A; Koshiya and Smith, 1999). Successful photolysis evoked a $\mathrm{Ca}^{2+}$ signal in targeted neurons, consistent with firing of multiple APs (Fig. 3A; Morgado-Valle et al., 2008), that could be blocked by $25 \mu \mathrm{M}$ NBQX, an AMPA receptor antagonist (Fig. 3A). Since the same stimulus parameters could elicit different neuronal responses across slices, we raised power from $1 \mathrm{~mW}$ until a $\mathrm{Ca}^{2+}$ response was observed in stimulated neurons, indicating that a burst of APs was initiated. This laser power (1-3 mW) was in a range to produce a burst of APs only in the targeted neuron and served as an upper bound for laser power/spot for the remainder of the experiment. Successful evoked preBötC bursts were determined by occurrence of a XIIn burst within $1 \mathrm{~s}$ of the stimulus. Since XIIn bursts could coincidentally fall within this time window (Fig. 4A), laser spots were delivered over 5-10 runs/trial at random phase in any given run.

The threshold number of targeted neurons that reliably (probability/trial $>80 \%$ ) generated an ectopic XIIn burst varied from slice to slice, ranging from 4 to $9(n=4$; Fig. $4 A, B)$. Evoked bursts did not differ significantly from endogenous bursts (Fig. $4 C$; amplitude, $t_{(12)}=-0.03, p=1$; half-width, $t_{(12)}=0.18, p=$ 0.9 , area, $\left.t_{(12)}=0.09, p=0.9 ; n=7\right)$. Shifting a holographic pattern with a suprathreshold number of spots 50-200 $\mu \mathrm{m}$ off their somatic targets reduced probability/trial of ectopic bursts from $98 \pm 2 \%$ (on-cell) to $15 \pm 8 \%$ (off-cell; $n=3$ ). Overlap of the shifted pattern with other rhythmogenic neurons or an endogenous burst coincident with the stimulus time window (Fig. $4 A$ ) could contribute to the nonzero probability of eliciting ectopic bursts in the off-cell condition.

Although targeted neurons generated APs within $20 \mathrm{~ms}$ of photolysis onset (Fig. 2), the onset of evoked XIIn bursts was delayed $255 \pm 43 \mathrm{~ms}$ when stimulating threshold numbers of neurons $(n=4)$. Latency was inversely related to the number of neurons stimulated. Occasional bursts elicited when stimulating subsets of the threshold pattern containing 1-3 fewer neurons (probability/trial $<80 \%$ ) were delayed $378 \pm 53 \mathrm{~ms}$, significantly longer than the latency for supersets of the threshold pattern containing $1-3$ additional neurons (125 \pm $23 \mathrm{~ms} ; t_{(3)}=5.1 ; p=0.01 ; n=4$; Fig. 4D). XIIn bursts could not be evoked when stimulating any threshold set of neurons within $500 \mathrm{~ms}$ after an endogenous burst (Fig. 4 E).
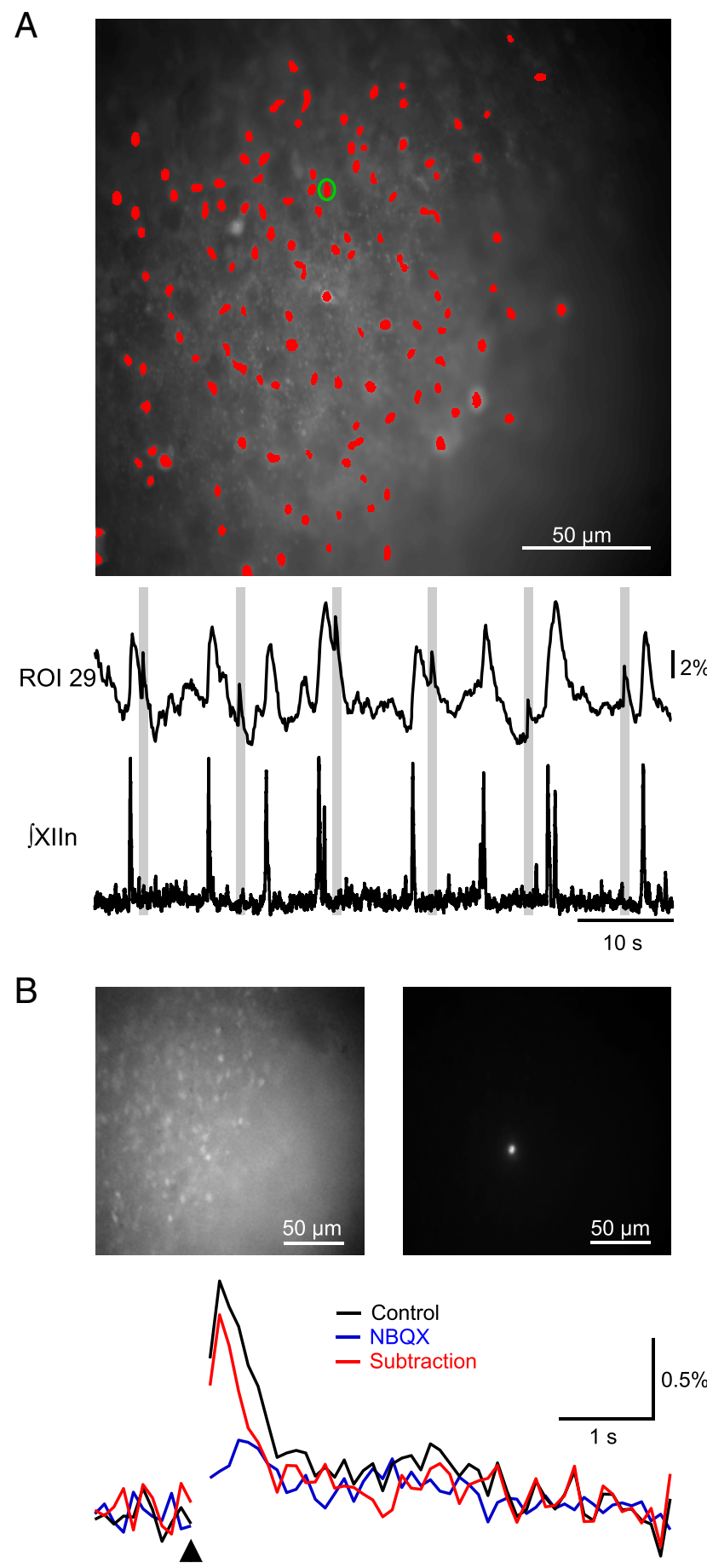

Figure 3. Holographic photostimulation evokes increases in $\mathrm{Ca}^{2+}$ fluorescence. $A$, Fluorescent image of $\mathrm{Ca}^{2+}$-dye loaded preBötC neurons with ROls marked in red and $\mathrm{ROI} 29$ marked with a green circle (top). Normalized fluorescence trace for ROI 29 (middle), which was stimulated with a single $10 \mu \mathrm{m}$ diameter, $3 \mathrm{~mW}$ spot at the times indicated by gray bars, and simultaneous electrophysiological recording (bottom) of endogenous XIIn activity during stimulation are shown. Stimulation of a single neuron did not trigger network bursts as indicated by the absence of activity in XIIn during laser stimulation (bottom), but did result in increases in $\mathrm{Ca}^{2+}$ fluorescence (middle). B, Average wide-field fluorescence (top, left) and contrastadjusted frame showing only the laser spot (top, right). Stimulation (black triangle) of single preBötC neurons produces fluorescence rises, which are almost completely blocked by $25 \mu \mathrm{M}$ NBQX (bottom). Subtraction trace represents difference between control and NBQX traces. Laser stimulus artifact is blanked. 
A
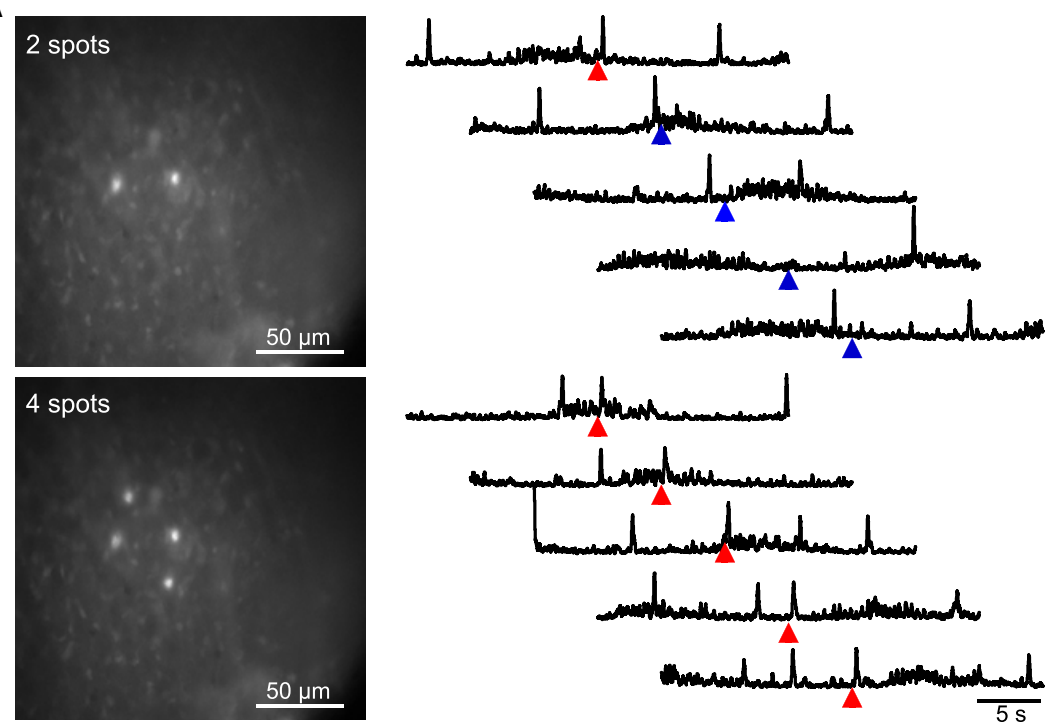

B
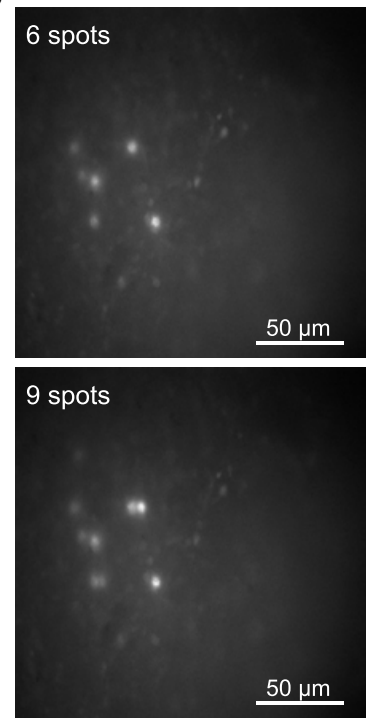
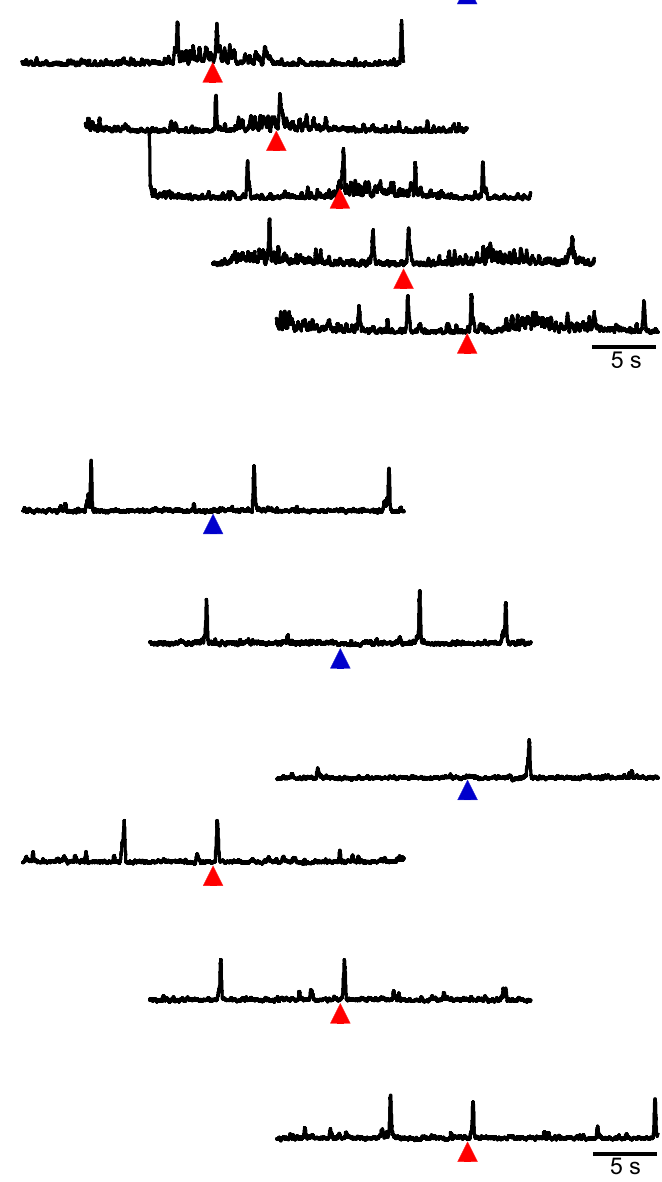

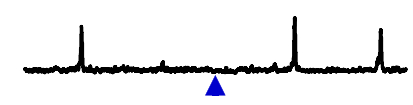

C

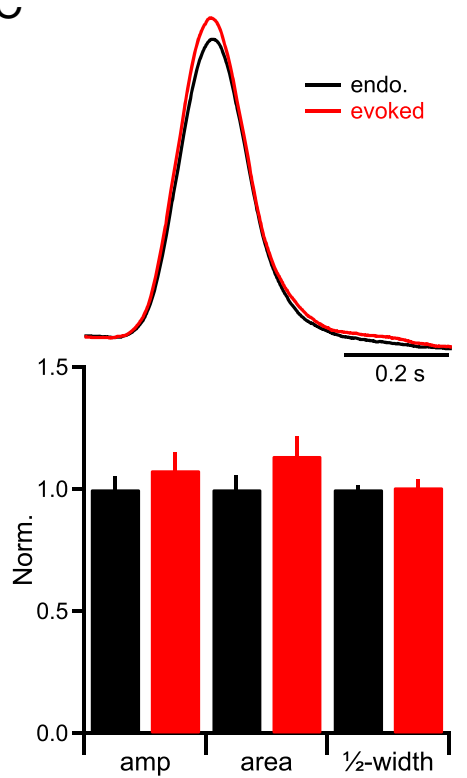

$\mathrm{D}$
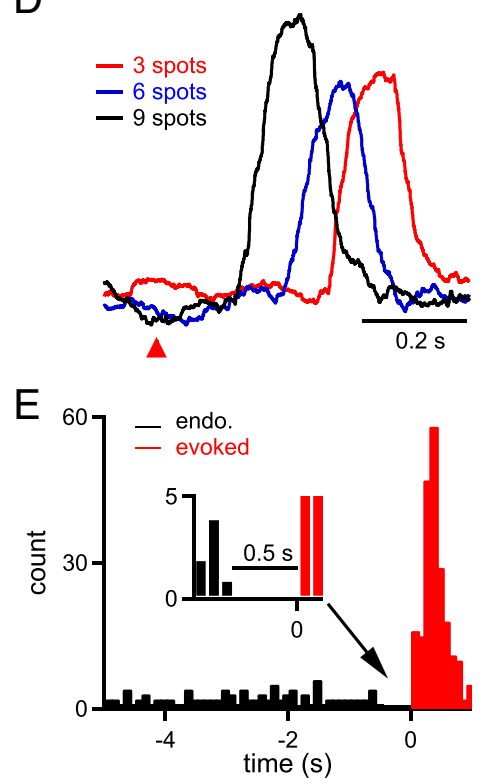

Figure 4. Targeting 4-9 inspiratory-modulated preBötC neurons triggers XIIn bursts. $A, B$, Representative examples from two slices in which the threshold number was 4 neurons (laser power $\approx$ $3 \mathrm{~mW} / \mathrm{spot}, 5 \times 800 \mu$ s pulses, $200 \mathrm{~Hz}$ ) $(\boldsymbol{A})$ or 6 neurons (laser power $\approx 1-3 \mathrm{~mW} / \mathrm{spot}, 5 \times 800 \mu$ s pulses, $200 \mathrm{~Hz}$ ) $(\boldsymbol{B})$ showing frame when stimulation was applied (left) and activity recorded from XIIn (right). Arrowheads depict when laser stimulation was applied with success (red triangle) and failure (blue triangle) for burst initiation. Sharp, large amplitude (amp), upward deflections represent XIIn bursts. C, Averaged trace (top) and group normalized (Norm.) data of burst shape parameters (bottom) show that evoked XIIn bursts do not differ from endogenous (endo.) XIIn bursts. D, Sample inspiratory bursts recorded in XIIn as a function of number of spots reveal a substantial delay between laser stimulation (red triangle) and burst generation. $\boldsymbol{E}$, Histogram showing burst times in all trials from $n=7$ slices where laser stimulation (at $t=0$ ) successfully evoked bursts. Laser-evoked (red) bursts and preceding endogenous (black, control) bursts are depicted. A refractory period of $0.5 \mathrm{~s}$ following an endogenous burst, where laser stimulation could not trigger motor output, was apparent (inset).

\section{Discussion}

The brain is capable of exquisite control over motor behavior. Stimulation of single neurons in mammalian motor cortex can trigger complex rhythmic movements (whisking) by activation of a central pattern generator (CPG) (Brecht et al., 2004). However, the number of neurons that must be activated within CPGs to trigger motor output has not been determined for any mammalian motor behavior. The threshold behavior of the preBötC, determined here with holographic photolysis, places critical and novel boundary conditions on the microcircuit underlying inspiratory burst generation.
The preBötC neural circuit can amplify the activity of a few neurons, $<1 \%$ of the population, into a burst of $\sim 1000$ neurons. The inability of a single neuron to initiate network bursts may ensure regularity in respiratory rhythm generation, as inputs or stochastic fluctuations leading to APs in a single neuron would be insufficient to prematurely generate an inspiratory burst. Requiring simultaneous or overlapping bursting in a small number of neurons for initiation of XIIn output allows for rapid adaptability, crucial in a network that must constantly and sometimes rapidly adjust to changing metabolic demands and accommodate overlapping volitional, 
emotive, and reflexive behaviors (Feldman and Del Negro, 2006; Feldman et al., 2013). Elucidating preBötC network mechanisms that support this threshold may improve our understanding of disorders of the nervous system that destabilize the breathing rhythm, such as Rett syndrome, Parkinson's, multiple-systems atrophy, and amyotrophic lateral sclerosis (Feldman and Del Negro, 2006; Feldman et al., 2013).

By empirically determining responses to glutamate diffusion and comparing the effective volume of laser illumination with preBötC neuron distributions, we determined that only the targeted neuron would be sufficiently stimulated to generate a burst of APs and propagate activity through the network. Glutamate diffusion away from the focused laser spot may influence additional neurons; however, with a rheobase of $32 \mathrm{pA}$, these neurons would likely not reach AP threshold, and activation would be limited to subthreshold depolarization (Rekling et al., 2000). Subthreshold depolarization could propagate through gap junctions; however, the weak coupling coefficient between preBötC neurons would strongly attenuate any response (Rekling et al., 2000). The significant decrease in the probability of (evoked) burst generation when the pattern is displaced also (empirically) suggests that incidental illumination does not have a large effect. While dendritic responses fell below rheobase, amplifying conductances in dendrites, e.g., $\mathrm{Ca}^{2+}$-activated nonspecific cation current $\left(\mathrm{I}_{\mathrm{CAN}}\right)$ or persistent $\mathrm{Na}^{+}$current $\left(\mathrm{I}_{\mathrm{NaP}}\right)$, could have been affected by the use of TTX in these experiments. However, presumptive dendritic $\mathrm{I}_{\mathrm{CAN}}$ activation, even from multiple sites, does not always elicit somatic action potentials (Del Negro et al., 2011, their Fig. $2 H$ ).

Burst initiating neurons are unlikely to be restricted to a small, specialized class of preBötC neurons, since our targets were randomly selected based solely on their inspiratorymodulated activity and the threshold number of targets was small. The broad criteria for target selection may also overestimate the threshold number, as a subset of glycinergic preBötC neurons are inspiratory-modulated (Winter et al., 2010). Since burst generation is a presumptively excitatory process, stimulation of inhibitory neurons could even detract from burst initiation. The capacity of potentially any excitatory inspiratory-modulated preBötC neuron to underlie burst initiation may protect respiration from catastrophic failure due to loss of any specific single (or set of) neuron(s) from disease or aging (Feldman and Del Negro, 2006; Hayes et al., 2012; Feldman et al., 2013).

Network properties facilitating population bursting, such as the degree or strength of connectivity, must be reconciled with the significant delay observed between stimulation and burst initiation. This latency exceeds reasonable estimates of time for oligosynaptic propagation of activity. In a simple simulation of synaptic propagation of activity, stimulation of $4-6$ neurons in a network of 50 point-conductance neurons taken without modification from Butera et al. (1999) with a connectivity of 0.13 (Rekling et al., 2000) triggered a network-wide burst with a latency of $\sim 100 \mathrm{~ms}$ (K. Kam, unpublished observations).

Whether the $255 \mathrm{~ms}$ delay to an ectopic burst involves processes active during endogenous rhythmogenesis is not known; however, this latency is similar in time scale to preinspiratory firing in preBötC neurons, a several hundred millisecond period of activity preceding inspiratory bursts that does not result in motor output (Rekling et al., 1996). Due to this similarity, we speculate that photostimulation engages an obligatory process present during endogenous rhythmogen- esis and necessary for burst initiation. Processes capable of producing such a delay include the following: (1) depolarizing conductances with slow ( $>100 \mathrm{~ms}$ ) activation or inactivation kinetics in downstream neurons, e.g., $\mathrm{I}_{\mathrm{NaP}}$ or $\mathrm{I}_{\mathrm{CAN}}$ (Del Negro et al., 2005); (2) second messenger signaling pathways, perhaps initiated by dendritic $\mathrm{Ca}^{2+}$ (Morgado-Valle et al., 2008; Del Negro et al., 2011); (3) percolation of activity through a sparsely or weakly connected preBötC network (Rekling et al., 2000; Carroll and Ramirez, 2013); and/or (4) competing inhibitory/hyperpolarizing conductances, signaling pathways, or neurons (Feldman and Del Negro, 2006; Feldman et al., 2013). Determining which mechanisms underlie this delay, within the constraints set by other parameters of the threshold behavior, is critical for understanding preBötC burst generation and neural control of respiration. Patterned photostimulation represents a powerful method for elucidating these mechanisms and, more generally, for deconstructing active networks. Novel multi-neuronal perturbations can be used to explore connectivity and recapitulate patterns of activity in groups of neurons to reveal fundamental dynamics in neural circuits underlying behavior.

\section{References}

Brecht M, Schneider M, Sakmann B, Margrie TW (2004) Whisker movements evoked by stimulation of single pyramidal cells in rat motor cortex. Nature 427:704-710. CrossRef Medline

Butera RJ Jr, Rinzel J, Smith JC (1999) Models of respiratory rhythm generation in the pre-Bötzinger complex. II. Populations of coupled pacemaker neurons. J Neurophysiol 82:398-415. Medline

Carroll MS, Ramirez JM (2013) The cycle-by-cycle assembly of respiratory network activity is dynamic and stochastic. J Neurophysiol. Advance online publication. Retrieved September 19, 2012. doi:10.1152/ jn.00830.2011. CrossRef Medline

Del Negro CA, Morgado-Valle C, Hayes JA, Mackay DD, Pace RW, Crowder EA, Feldman JL (2005) Sodium and calcium current-mediated pacemaker neurons and respiratory rhythm generation. J Neurosci 25:446-453. CrossRef Medline

Del Negro CA, Kam K, Hayes JA, Feldman JL (2009) Asymmetric control of inspiratory and expiratory phases by excitability in the respiratory network of neonatal mice in vitro. J Physiol 587:1217-1231. CrossRef Medline

Del Negro CA, Hayes JA, Rekling JC (2011) Dendritic calcium activity precedes inspiratory bursts in preBötzinger complex neurons. J Neurosci 31:1017-1022. CrossRef Medline

Feldman JL, Del Negro CA (2006) Looking for inspiration: new perspectives on respiratory rhythm. Nat Rev Neurosci 7:232-242. CrossRef Medline

Feldman JL, Del Negro CA, Gray PA (2013) Understanding the rhythm of breathing: so near, yet so far. Annu Rev Physiol. Advance online publication. Retrieved January 19, 2013. doi:10.1146/annurev-physiol-040510130049. CrossRef Medline

Golan L, Reutsky I, Farah N, Shoham S (2009) Design and characteristics of holographic neural photo-stimulation systems. J Neural Eng 6:066004. CrossRef Medline

Hayes JA, Wang X, Del Negro CA (2012) Cumulative lesioning of respiratory interneurons disrupts and precludes motor rhythms in vitro. Proc Natl Acad Sci U S A 109:8286-8291. CrossRef Medline

Koshiya N, Smith JC (1999) Neuronal pacemaker for breathing visualized in vitro. Nature 400:360-363. CrossRef Medline

Lutz C, Otis TS, DeSars V, Charpak S, DiGregorio DA, Emiliani V (2008) Holographic photolysis of caged neurotransmitters. Nat Methods 5:821-827. CrossRef Medline

McCrimmon DR, Feldman JL, Speck DF (1986) Respiratory motoneuronal activity is altered by injections of picomoles of glutamate into cat brain stem. J Neurosci 6:2384-2392. Medline

Morgado-Valle C, Beltran-Parrazal L, DiFranco M, Vergara JL, Feldman JL (2008) Somatic $\mathrm{Ca}^{2+}$ transients do not contribute to inspiratory drive in preBötzinger Complex neurons. J Physiol 586:4531-4540. CrossRef Medline

Mukamel EA, Nimmerjahn A, Schnitzer MJ (2009) Automated analysis 
of cellular signals from large-scale calcium imaging data. Neuron 63: 747-760. CrossRef Medline

Rekling JC, Champagnat J, Denavit-Saubié M (1996) Electroresponsive properties and membrane potential trajectories of three types of inspiratory neurons in the newborn mouse brain stem in vitro. J Neurophysiol 75:795-810. Medline

Rekling JC, Shao XM, Feldman JL (2000) Electrical coupling and excitatory synaptic transmission between rhythmogenic respiratory neurons in the preBötzinger Complex. J Neurosci 20:RC113. Medline

Ruangkittisakul A, Okada Y, Oku Y, Koshiya N, Ballanyi K (2009) Fluorescence imaging of active respiratory networks. Respir Physiol Neurobiol 168:26-38. CrossRef Medline

Ruangkittisakul A, Panaitescu B, Ballanyi K (2011) $\mathrm{K}^{+}$and $\mathrm{Ca}^{2+}$ dependence of inspiratory-related rhythm in novel "calibrated" mouse brainstem slices. Respir Physiol Neurobiol 175:37-48. CrossRef Medline

Smith JC, Ellenberger HH, Ballanyi K, Richter DW, Feldman JL (1991) PreBötzinger Complex: A brainstem region that may generate respiratory rhythm in mammals. Science 254:726-729. CrossRef Medline
Tan W, Janczewski WA, Yang P, Shao XM, Callaway EM, Feldman JL (2008) Silencing preBötzinger Complex somatostatin-expressing neurons induces persistent apnea in awake rat. Nat Neurosci 11:538 540. CrossRef Medline

Tan W, Pagliardini S, Yang P, Janczewski WA, Feldman JL (2010) Projections of preBötzinger Complex neurons in adult rats. J Comp Neurol 518:1862-1878. CrossRef Medline

Valmianski I, Shih AY, Driscoll JD, Matthews DW, Freund Y, Kleinfeld D (2010) Automatic identification of fluorescently labeled brain cells for rapid functional imaging. J Neurophysiol 104:1803-1811. CrossRef Medline

Winter SM, Fresemann J, Schnell C, Oku Y, Hirrlinger J, Hülsmann S (2010) Glycinergic interneurons in the respiratory network of the rhythmic slice preparation. Adv Exp Med Biol 669:97-100. CrossRef Medline

Zahid M, Vélez-Fort M, Papagiakoumou E, Ventalon C, Angulo MC, Emiliani V (2010) Holographic photolysis for multiple cell stimulation in mouse hippocampal slices. PLoS One 5:e9431. CrossRef Medline 